\title{
A Compositional Spatio-temporal Semantics for French Motion Verbs and Spatial PPs.
}

\author{
Nicholas Asher and Pierre Sablayrolles \\ IRIT, University Paul Sabatier \\ Toulouse, France
}

\section{Introduction}

This paper yields a semantics for the spatio-temporal properties of motion complexes in French. By motion complex we understand here a motion verb followed by a spatial prepositional phrase adjunct (PP). Unlike other semantic or syntactic studies, we build up the spatio-temporal semantic properties of motion verb complexes compositionally, on the basis of the semantic properties of the verb, its arguments and adjuncts in the complex. We claim that the spatial and temporal semantics of a motion verb complex is a specifiable function of the spatial and temporal semantics of each component of the complex. Let us illustrate this by contrasting the complexes given in the following examples.

(1) a. sortir du jardin

to go out of the garden

b. sortir par le jardin

to go out through the garden

c. sortir dans le jardin

to go out into the garden

d. s'éloigner dans la rue

to go away on the street

In the three sentences (1a), (1b) and (1c), the same motion verb sortir and the same location denoting noun phrase le jardin is used. These complexes differ only in the preposition used to link the location in the PP to the motion verb. In (1a), the complex requires that the mobile (the entity which moves) is inside the garden before the process begins and outside after it terminates; in (1b), the complex requires that the mobile is inside some location before the process begins, outside it (ie. inside another location) after the process ends, and that it must pass through the garden during the moving process; in (1c), the complex requires that the moving entity is inside some location, which is not the garden before the process begins and inside the garden afterwards.

In (1a), (1b) and (1c), we have in changing just one component of the complex seen that the meaning of the complex has completely changed. But of course we can vary all the components of the meaning complex. Compare for example (1a) and (1d). We need to be able to calculate the meanings of such complexes in a rule-governed way.

(C) 1994 by Nicolas Asher and Pierre Sablayrolles

Mandy Harvey and Lynn Santelmann (eds.), SALT IV 1-15, Ithaca, N.Y.: Comell University. 
To capture the variations in meaning seen above, we will offer a typology of motions verbs and spatial prepositions that can make up motion complexes in French. We distinguish between motion verbs and motion verb complexes; the motion verbs contribute but do not completely determine a motion complex. The compositional rules we propose here make this contribution precise.

\section{Ontology and Semantic Framework}

The typology of motion verbs we detail below considers motion verbs without any adjuncts, independently of any tense or mode and without reference to any particular context. We do this in order to extract their, and only their, intrinsic semantics. To be sure even this requires certain ontological assumptions about processes, objects, boudaries and the like. We are interested here in a typology of motion as it is expressed in natural language, that is motion is a property of objects (ie. objects move), but motions themselves are a type of event. Motions are changes of some sort of spatial position over time. To explore motion, we first make clear our ontological assumptions concerning eventualities and spatiotemporal extensions.

Eventualities are concrete but complex objects. They may have both objects as constituents and also other eventualities. Following [Vie91], we assume that every concrete entity has a spatio-temporal trajectory, which we will denote with the aid of a function STref. STref(x) is the spatio-temporal extent of $x$. Our semantic framework for representing about spatio-temporal expressions will be Discourse Representation Theory (or DRT) of [Kam79]. In DR-theoretic terms, STref(x) is a new discourse entity designating the trajectory described by $\mathrm{x}$ all along its "life". STref may be additionally parametrized with the aid of a temporal variable: STref(x,e) denotes the "temporal slice" of STref(x) whose time matches the time of the event $e$, if e is temporally included in $\operatorname{STref}(x)$; otherwise it is not defined. We will assume here that we can compare $\operatorname{STref}(x, t)$ with $\operatorname{STref}\left(x, t^{\prime}\right)$ for $t$ $\neq \mathrm{t}^{\prime}{ }^{1}{ }^{1}$ Spatio-temporal relations between these referents, such as inclusion $(\mathrm{P}(\mathrm{x}, \mathrm{y}))$, overlap $(\mathrm{O}(\mathrm{x}, \mathrm{y}))$ or contact $(\mathrm{EC}(\mathrm{x}, \mathrm{y}))$ are axiomatized in a theory of space-time, derived from Clarke's calculus of individuals ([Cla81] and [Cla85]), and which is based on mereology [Les27-31]. This theory, fully presented in [Aur91] and [Vie91], is used in [AV93] for representing the geometrical aspects of the lexical semantics of static relations such as être dans (to be in), être sur (to be on), être devant (to be in front of), and internal localization nouns such as le haut (the top), le dessus (the top surface), le coin (the comer) ... It also can be used to encode various facts about the geometrical and topological properties of objects and processes or, rather, their STref projections (cf. [Vie91]). We will suppose, for simplicity, that objects of the sort $\operatorname{STref}(\mathrm{x})$ obey the laws of $\mathrm{R}^{4}$ and have a regular, metric topology.

Along with localists like [Jac76], we further suppose that all eventualities have a beginning or source, an end or goal, and a middle or path. We hold that these parts of eventualities are an aspect of "natural language metaphysics" Source(e) is a "location" whose spatio-temporal referent contains or is in contact with STref(e,Init(e)), and similarly for Goal(e). Finally, Path(e) will be a set of sequences of "locations" $l_{i}$. A path is a set of sequences because we need to allow for descriptions at different granularities, and this is necessary to handle the 
interactions between our lexical semantics for motion complexes and discourse structure (see [AS94], [Ash-a193]).

For each sequence of locations $\left\langle\mathrm{l}_{1}, \ldots, \mathrm{l}_{\mathrm{n}}\right\rangle$, we have $\mathrm{P}\left(\operatorname{STref}(\mathrm{e}), \Sigma_{\mathrm{i}}\right.$ STref $\left.\left(l_{i}\right)\right)$, and for each $i, \operatorname{EC}\left(\operatorname{STref}\left(l_{i}\right), S \operatorname{STref}\left(l_{i+1}\right)\right)$, ie. adjacent locations in the sequence are externally connected.

Source(e) and Goal(e) are not sets of "locations", however. They are often lexicalized, thus uniquely identified within the discourse by a particular discourse referent introduced by the noun phrase; in other cases they are anaphorically identified exploiting constraints introduced by discourse structure [Ash94], and in the remaining cases their existence is assumed through the introduction of discourse referents. Our theory differs from that of other localists in that we exploit discourse structure and the spatiotemporal properties of the objects introduced in a text to determine these locations.

Our study also requires us to make explicit our assumptions concerning the semantics of motion expressions. We will follow roughly the approach of Davidson [Dav67] (an approach which is also used in DRT), on which eventualities, along with other objects, satisfy predicates derived from natural language verbs. Our general approach will be to import the toplogical and mereological formalism of ([Les27-31], [Cla81] and [AV93]) into a particular semantic formalism--that of DRT (though were we not interested in problems of anaphora resolution and discourse structure, we could have chosen another formalism). Thus, motion complexes will for us be represented by DR-theoretic structures that correspond to the contribution of a VP and which will describe an eventuality. In the bottom up construction procedure for building DRSs in [Ash93], intransitive verbs will introduce a predicative $D R S$ or dynamic relation between an individual and an eventuality. Such a predicative DRS serves as an argument to a partial DRS which is introduced either by a determiner or the inflection node; partial DRSs are dynamic quantifiers that serve to introduce discourse referents into DRtheoretic structures, and it is these discourse referents that serve as arguments to predicative DRSs. ${ }^{2}$ The eventuality discourse referents themselves are introduced by the inflection node in the syntactic tree along with certain information concerning the tense of the verb. Such eventuality discourse referents also are arguments to various adverbial phrases, and in particular, spatial prepositional phrases, which have the form $\lambda P \lambda \mathrm{e}^{\prime}\left[\mathrm{U},\left\{P\left(\mathrm{e}^{\prime}\right), \mathrm{A}\left(\mathrm{e}^{\prime}\right)\right\}\right]$ where $\mathrm{U}$ is a set of discourse referents, $P$ is a variable for dynamic properties derived from VPs. Thus, prepositional phrases also introduce DR-theoretic structures that like predicative DRSs need discourse referents to become full fledged DRSs but that like partial DRSs take properties as arguments. These structures are built up by application of the DR-theoretic structure for the preposition in the head of the PP and the partial DRS derived from the noun phrase in the complement, and in general DRSs are built by means of this application. ${ }^{3}$ Due to font limitations imposed by the SALT volume editors, we cannot unfortunately represent detailed examples of the DRS construction procedure. The interested reader should consult [AS94].

One of our tasks in this paper is to classify the various eventuality discourse referents that might be understood as representing movements and to determine those inferences that allow us to specify the spatio-temporal position of the eventuality as a whole or of its parts or actors with respect to various reference locations given in a text. In this paper we will present both a typology and rules for drawing inferences about the locational structure. 


\section{The 4 Classes of Motion Verbs}

Following Gruber [Gru65], Jackendoff [Jac76], Boons [Boo85], Guillet [Gui90], ..., we approach motion verbs in terms of some "localist semantical" role labels. We specify the basic semantics of motion verbs and prepositions in terms of their relations to three types of spatio-temporal entities : locations, positions, and postures :

A Location is a portion of space 4 which can be "designated" in natural language, and with which is associated a functionality. Locations are generally lexicalized by means of a real lexical item (eg. la cuisine (the kitchen)) or a construction using a preposition and a real lexical item (eg. derrière la maison (behind the house)). They can also be expressed by means of deictic constructions like ici (here) or là où Jean était il y a 5 minutes (where John was 5 minutes ago).

A Position is a portion of space within a location, without any associated functionality or proper lexical item ${ }^{5}$, and only geometrically defined by the pragmatic shape (see the definition below) associated with a given entity. A position thus is necessarily dependent on the entity used for its definition.

A Posture is a special way to be inside one's pragmatic shape (see the definition below), with which is associated a certain functionality. Postures are always postures of an entity; they are defined by the relations between the parts of that entity. Postures are lexicalized by participial forms of verbs of changes of postures - eg. assis (sitting down), or by adjectives - eg. debout (standing up).

By pragmatic shape we mean the 3-D portion of space fully occupied by the entity, plus that space that would be occupied by the entity $\mathrm{x}$ were it to undergo $\mathrm{a}$ change of posture $p$, where $p$ is constrained such that if $p$ occurs from $t$ to $t$ and posture $(x, t)=\operatorname{posture}\left(x, t^{\prime}\right)$, then $\operatorname{STref}(x, t)$ is at least roughly the same as $\operatorname{STref}\left(\mathrm{x}, \mathrm{t}^{\prime}\right)$.

These ontological categories will allow us to avoid certain difficulties in earlier studies (e.g. [Boo85]) with the concept of "location," which was not properly defined. For instance, we will be able to distinguish the spatio-temporal meanings of the minimal pairs entrer (to go in) - sortir (to go out) on the one hand and sortir (to go out) - partir (to leave) on the other. Further, our ontological assumptions will allow us to compute the right meanings for the motion complexes - sortir de/par le/dans le jardin (to go out of/by/into the garden).

We can now specify the concepts of Source, Path and Goal, introduced in the previous section, with each of our location, position and posture. Source(e), Initposition(e) and Init-posture(e) are a location, position and posture, respectively, whose spatio-temporal referent contains or is in contact with STref(e,Init(e)); we define Goal(e), Final-position(e) and Final-posture(e) analogously. Finally, Path(e) will be a set of sequences of locations and Path-position(e) and Path-posture(e) will be a sequence of positions and postures, respectively.

For us a motion verb involves a change in either the location, position or posture of one or more objects (though for our study we have restricted ourselves to 
contexts with one mobile). To be a little more precise, we define four ${ }^{6}$ classes of motion verbs:

- the verbs of change of location (CoL), which entail that the mobile changes location during the process. Members of this class are, for example, entrer (to enter), arriver (to arrive).

- the verbs of change of position (CoPs), which entail that the mobile changes position during the process. Members of this class are, for example, se déplacer (to move around), circuler (to circulate).

- the verbs of inertial change of position (ICoPs), which imply, by default, a change of position for the mobile. Members of this class are, for example, courir (to run), voler (to fly); they can combine with the adverbial in place, in contrast with CoPs verbs.

- the verbs of change of posture (CoPtu), which entail that the mobile stays in the same location and at the same position during the whole process, but also changes of posture during the process. Members are, for example, s'asseoir (to sit down), se baisser (to bend down).

The 3 first classes are not exclusive; an eventuality described by CoL verb may also be described by CoP verb; and any eventuality described by a CoP verb may also be described by an ICoPs verb. One cannot change location without changing position; and one cannot change position without inertially changing position. In contrast, one can change location or position without changing posture. These classes also serve to characterize motion complexes. But in view of the relationships between the classes, the addition of arguments and adjuncts to a motion verb either does not affect the classification of the resulting complex (i.e. it falls in the same class as the motion verb it contains) or it forces the resulting complex to belong to one of the above described subclasses of the class of the motion verb.

\section{A Conceptual Structuration of Space}

In the following of this paper we focus on CoL (Change of Location) verbs and $\mathrm{CoL}$ complexes, and offer a more detailed analysis for them. CoL verbs are propicious to a detailed analysis of how the space is organized in and around the location with respect to which the displacement take sense. They describe a displacement going from outside this location to the inside of it, or the reverse displacement. Nevertheless, a coarse organization of the space in only two "zones", the interior and the exterior of the location, quickly proves insufficient ([Boo85], [Lau91]). Compare for example (2a) with (2b) and (3a) with (3b).

(2) a. Abby est sortie de la maison

Abby has gone out of the house

b. Abby est partie de la maison

Abby has gone away from the house 
(3) a. L'avion a atterri sur la piste 4

The plane has touched down on runway 4

b. L'avion s'est approché de la piste 4

The plane has approached runway 4

In both (2a) and (2b), Abby has gone from the inside of the house to the outside of it. But if sortir in (2a) only describes this displacement, partir in (2b) forces Abby to continue her displacement till she is away from the house, at a sufficient distance. Under a certain critical distance, the verb partir could not be used. To treat such verbs, we distinguish between an outside of proximity (called Z-outer-halo for Zone of outer halo) and a faraway outside (called Z-outer-most for Zone of outer most).

With (3a) and (3b), the plane stays, during its whole motion, outside the location which here is runway 4 . Nevertheless, the displacement is not the same in the two sentences. In (3a), the plane touches down and thus finally comes in contact with the runway, whereas in (3b) it has approached the runway without touching it. To take this into account, we introduce an external zone of contact (called Z-contact).

We finally arrive to the following conceptual structuration of space (fig. 1), with respect to a location of reference (lref), which on our approach is either determined by the NP in the prepositional phrase or identified anaphorically with a location introduced in earlier discourse:

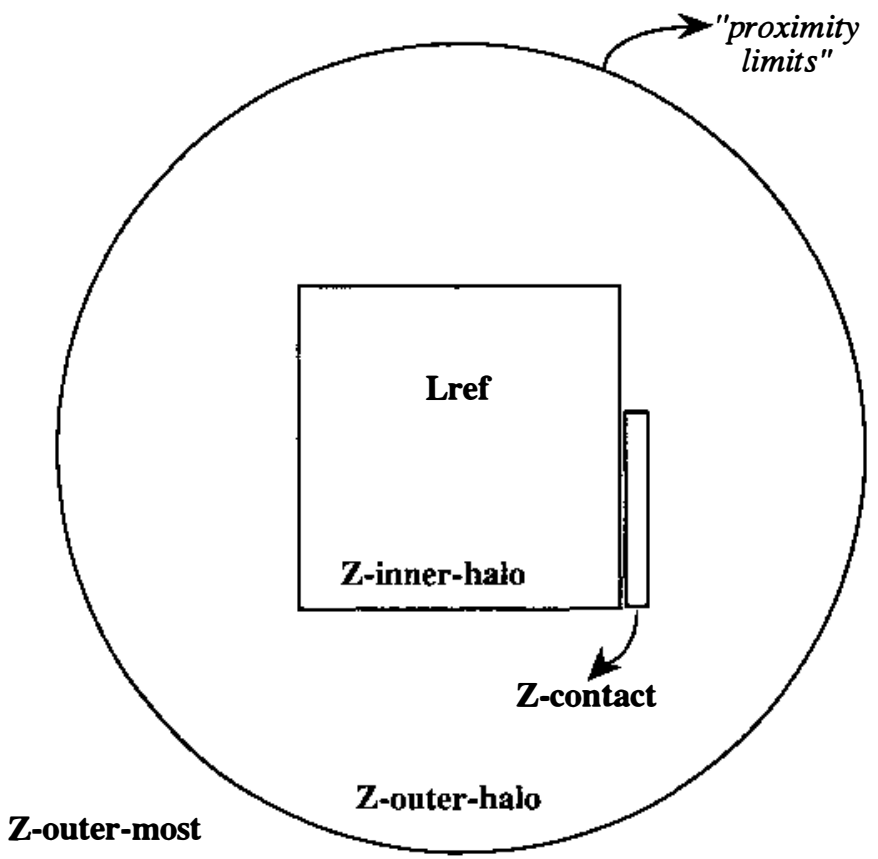

Figure 1 : The Conceptual Structuration of Space into 4 Zones 


\section{The Subclassification of CoL Verbs}

Our class of CoL verbs can be refined on the basis of which zones the moving entity is inside, at the begining and at the end of its motion. All the possibilities are not lexicalized in French. In a systematic linguistic study we have found 216 lexical entries for French intransitive CoL verbs that have allowed for the definition of the 10 following subclasses, illustrated on fig. 2. We describe these classes here informally (see [AS94] for the formal details).

- the S'approcher (to approach) class : the mobile goes from Z-outer-most to Z-outer-halo, ie. from a far away outside to a near outside of a location of reference. Examples are : S'approcher (to approach); s'avancer (to move forward); accourir (to rush up ).

- the Arriver (to arrive ) class : the mobile goes from Z-outer-most to Z-innerhalo, via Z-outer-halo. Examples are : arriver (to arrive ); aller (to go ); venir (to come ).

- the Entrer (to enter) class : the mobile goes from Z-outer-halo to Z-innerhalo. Examples are : entrer (to enter); s'embarquer (to board); pénétrer (to penetrate ).

- the Se Poser (to land) class : the mobile goes from Z-outer-halo to Z-contact. Examples are : se poser (to land); se jucher (to perch); se suspendre (to hang ).

- the S'éloigner (to distance oneself from ) class : the mobile goes from Z-outerhalo to Z-outer-most. Examples are : s'éloigner (to distance oneself from ); s'isoler (to become isolated); se reculer (to move back).

- the Partir (to leave ) class : the mobile goes from Z-inner-halo to Z-outermost, via Z-outer-halo. Examples are : partir (to leave ); s'en aller (to go away); déserter (to desert).

- the Sortir (to go out ) class : the mobile goes from Z-inner-halo to Z-outerhalo. Examples are : sortir (to go out); débarquer (to land); jaillir (to spring (up) ).

- the Décoller (to take off) class : the mobile goes from Z-contact to Z-outerhalo. Examples are : décoller (to take off); se décoller (to come unstuck); déconnecter (to disconnect).

- the Passer (par) (to go through ; to cross) class : the mobile goes from Zouter-halo, enters the location of reference (ie. Z-inner-halo), crosses it, and goes outside to Z-outer-halo. Examples are : passer (par) (to go through; to cross ); couper (to cross); repasser (par) (to pass by again ).

- the Dévier (to deviate) class : the verbs of this class describe the same kind of displacement as the ones of the Sortir class, except that Z-inner-halo and Z- 
outer-halo are here relative to an ideal trajectory ${ }^{7}$ instead of a "real" location of reference.
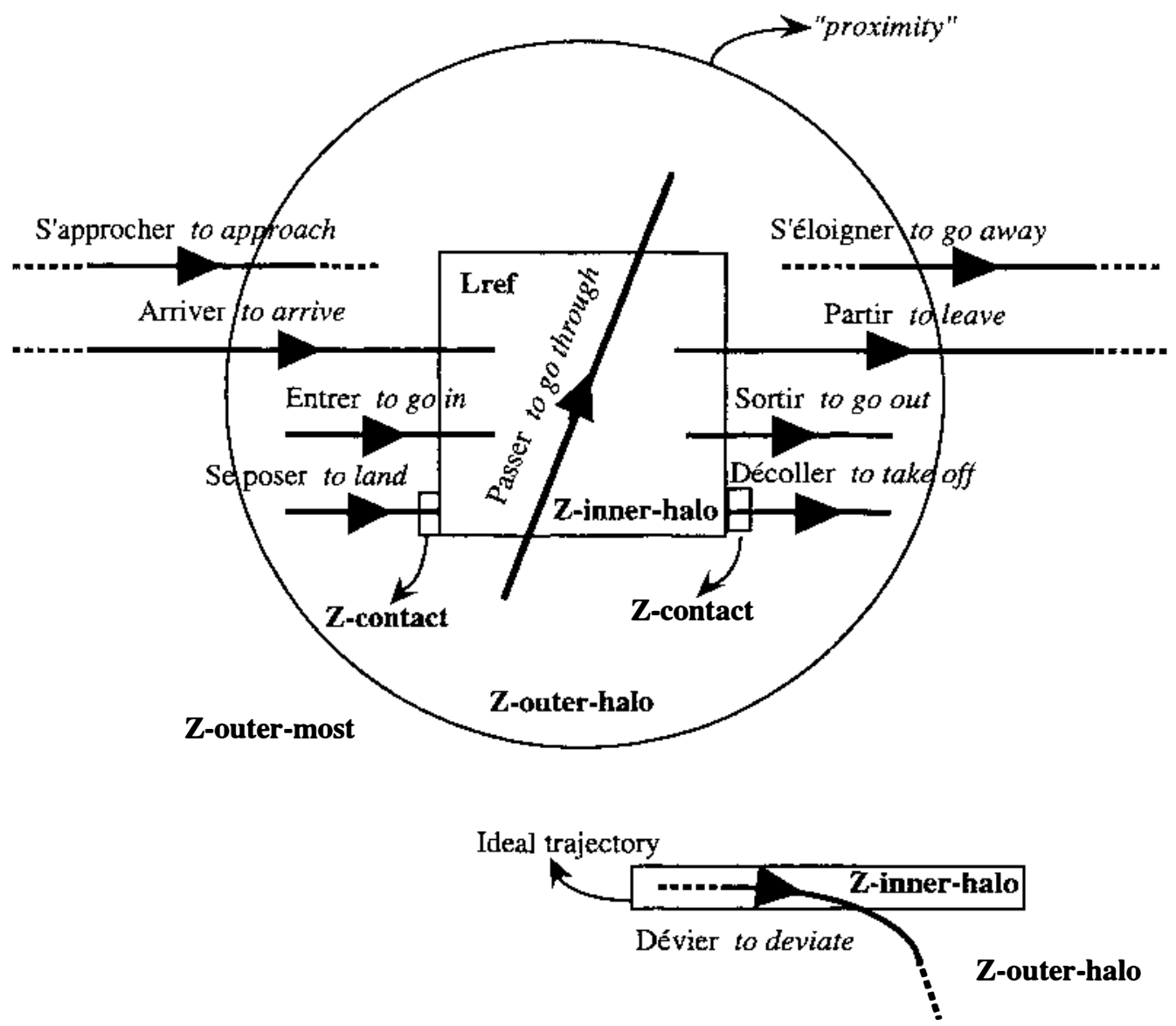

Figure 2 : The 10 Groups of CoL Verbs

Further, CoL verbs are classified as either Initial, Medial or Final. Final verbs focus on the goal, Medial on the path and Initial on the source. The classes S'éloigner, Partir, Sortir, Décoller and S'écarter have an initial polarity. The classes S'approcher, Arriver, Entrer and Se Poser have a final polarity. The class Passer (par) has a medial polarity.

\section{The Classification of Spatial Prepositions}

We have followed the same approach with spatial prepositions. Following [Lau93], we consider simple prepositions, like dans (in) as well as prepositional phrases, like en face de (in front of). We have classified 199 such French prepositions into the 16 following groups (see fig. 3) using in addition of our zones 
two other criteria: prepositions can be positional (in) or directional (into); directional prepositions can be Initial, like de (from), Medial, like par (through) or Final, like vers (towards), depending if they focus on the source, the path or the goal.

\begin{tabular}{|c|c|c|c|c|}
\hline Preposition & Z-inner-halo & Z-contact & Z-outer-halo & Z-outer-most \\
\hline Positional & $\begin{array}{c}\text { chez; dans } \\
\text { at; in }\end{array}$ & $\begin{array}{c}\text { sur; contre } \\
\text { on; against }\end{array}$ & $\begin{array}{c}\text { sous; derrière } \\
\text { below; behind }\end{array}$ & $\begin{array}{c}\text { loin de } \\
\text { far away from }\end{array}$ \\
\hline $\begin{array}{c}\text { Initial } \\
\text { Directional }\end{array}$ & $\begin{array}{c}\text { de chez } \\
\text { from_'s }\end{array}$ & $\begin{array}{c}\text { de sur } \\
\text { from onto }\end{array}$ & $\begin{array}{c}\text { de derrière } \\
\text { from behind }\end{array}$ & $\begin{array}{c}\text { de dehors } \\
\text { from the } \\
\text { outside }\end{array}$ \\
\hline $\begin{array}{c}\text { Medial } \\
\text { Directional }\end{array}$ & $\begin{array}{c}\text { par } \\
\text { through }\end{array}$ & au fil de & $\begin{array}{c}\text { le long de } \\
\text { along }\end{array}$ & $\begin{array}{c}\text { au-delà de } \\
\text { beyond }\end{array}$ \\
\hline $\begin{array}{c}\text { Final } \\
\text { Directional }\end{array}$ & $\begin{array}{c}\text { jusque dans } \\
\text { up to the inside } \\
\text { of }\end{array}$ & $\begin{array}{c}\text { jusque sur } \\
\text { up onto }\end{array}$ & $\begin{array}{c}\text { vers } \\
\text { towards }\end{array}$ & $\begin{array}{c}\text { pour } \\
\text { for }\end{array}$ \\
\hline
\end{tabular}

Figure 3 : Classification of French Spatial Prepositions

\section{Rules of Compositional Semantics}

On the basis of these classifications, we offer compositional rules, calculating the spatio-temporal properties of the complex from the ones of the verb and the ones of the PP. These rules (more precisely these axioms) are expressed in a logical form and a particular logical language. Again because of the font limitations we mentioned earlier, we will not give any formal details here. If the reader is interested in these details, he should consult [AS94]. Below we give a discursive overview of what these rules look like, and illustrate them with three examples.

First of all, we have three general rules (rules 1, 2 and 3) which apply whatever motion verb is combined with whatever spatial preposition. They in fact concern only the verb and are used to deduce the way the source (rule 1), the path (rule 2) and the goal (rule 3) are connected with the location of reference. That is, if the verb belongs for example to the Arriver class, then we know from this class that it describes a motion going from a Z-outer-most to a Z-inner-halo, via a Z-outerhalo, each of these three zones beeing related to a same location of reference. The three axioms will consequently connect the source with Z-outer-most, the path with $\mathrm{Z}$-outer-halo and the goal with Z-inner-halo, using a relation of spatio-temporal inclusion ${ }^{8}$.

Then, we have four rules (rules 4, 5, 6 and 7) which apply only when the spatial preposition involved in the complex is a directional one. Since directional prepositions intrinsically contain, as do CoL verbs, a direction of motion, we have two possibilities when we combine such a preposition with a motion verb. Either the verb and the preposition are in a relation of congruence (rule 4), ie. have the same polarity (initial, medial or final), or they are in a relation of non-congruence (rules 5, 6 and 7), i.e. have different polarity. In the first case, the verb and the preposition are "compatible" since they denote intrinsically the same behavior. The location introduced by the NP in the prepositional phrase can then be identified with the location of reference (introduced in the rules 1,2 and 3, but not instantiated yet). 
In the second case, the verb and the preposition are "incompatible"; in this case, it is the preposition which "dominates" and determines the source, path or goal. When the preposition has an initial polarity, it forces the source (rule 5) to be connected to the location introduced in the PP in its proper way too $0^{9}$; when it has a medial or final polarity, it forces the path (rule 6) or the goal (rule 7), respectively, to be connected to the location introduced in the PP in its proper way too. Consequently, the location of reference introduced by the rules 1,2 and 3 remains uninstantiated.

Finally, we have four rules (rules $8,9,10$ and 11 ) which apply only when the spatial preposition involved in the complex is a positional one. In constrast with directional prepositions, positional prepositions do not contain an intrinsic direction of motion. So there is no possible incompatibility with the motion verb concerning the determination of the lref. Nevertheless, there is sometimes an interesting interaction. When the motion verb has a final polarity (rule 11) or a medial polarity (rule 9), the verb specifies the lref to be a function of the meaning of the preposition applied to the denotation of the NP in the PP. The rule 9 gives us a "medial" reading of a complex like passer dans le jardin (pass through the garden)-- i.e., a reading in which the mobile takes a path that is within the street.Rule 9 says that the lref of the complex is a function of the meaning of the preposition applied to the denotation of the NP in the PP. On the other hand, medial verbs in French can combine with positional PPs to yield another reading. That is the directional reading of a complex like passer dans le jardin (to go into the garden). The mobile is outside the lref before its motion, and moves such a way that it finally enters the lref and is inside it at the end of its motion. Rule 10 says that in this case the PP specifies the goal of the motion complex and the lref, specified by the verb to include the path, remains uninstantiated. Finally, we have a rule (rule 8) for those motion complexes in which the verb has an initial polarity and the preposition is positional. In that case, the goal of the motion complex is specified by the NP in the PP and the lref, constrained by the verb to include the source, remains uninstantiated. A typical example is sortir dans la rue (to go out into the street). We can try to explain this puzzling behavior, if there exists some explanation, by saying that, for the French language, descriptions of displacement focus mainly on the path or the goal, and quite few on the source. For intransitive verbs, we have 85 verbs focusing on the source, 90 focusing on the path and 212 focusing on the goal. $95 \%$ of the transitive verbs focus on the path. So perhaps the language (or perhaps this is true for all languages and so is a feature of our natural language metaphysics) has a natural propensity to focus on final or medial parts of the eventuality described. This could explain why initial verbs are dominated by positional prepositions (rule 8), because this rule says that the complex has a final polarity-that is the complex focuses on the goal, whereas the verb focuses on the source and the preposition alone suggests no focus. The particularity of rule 10 is explained in another way. We remark that when the motion verb has a medial polarity, it can have two possible interpretations--the medial interpretation and final interpretation.

\section{Examples}

We now illustrate our rules with the three following examples:

(4) a. Jean est sorti dans le jardin John has gone out into the garden 


\author{
b. Jean est entré dans le jardin \\ John has gone into the garden \\ c. Jean est passé dans le jardin \\ John has gone through/into the garden
}

These 3 sentences use the same positional preposition (dans - in) which locates the theme inside the Z-inner-halo("garden ").

In (4a) we have the initial verb sortir (to go out), which belongs to the Sortir class. This verb denotes a displacement from the Z-inner-halo to the Z-outerhalo, with respect to a location of reference (lref). We here apply the rule 8 (Initial verb and positional preposition). This particular rule realizes a focus onto the goal and gives us a spatio-temporal relation of inclusion of the goal into the Z-innerhalo("garden"). The relation relates the goal with the location ("garden ") introduced in the PP instead of the location of reference because of the particularity of our rule 8 which allows the preposition to dominate the verb. The location of reference (lref) remains uninstantiated ${ }^{10}$. To conclude, we know that the source is part of the Z-inner-halo(lref) (rule 1), that the goal is part of the Z-outer-halo(lref) (rule 3) and that the goal is also part of the Z-inner-halo("garden ") (rule 8).

In (4b) we have the final verb entrer (to go in), which belongs to the Entrer class. This verb denotes a displacement from Z-outer-halo to Z-inner-halo, with respect to a location of reference. We here apply the rule 11 (Final verb and positional preposition). This rule tells us that the location of reference has to be matched with the zone Z-inner-halo("garden "). We then conclude that the source is part of the Z-outer-halo(Z-inner-halo("garden ")), which simplifies to the Zouter-halo("garden ") (using rules 1 and 11) and that the goal is part of the Z-innerhalo(Z-inner-halo("garden ")), ie. of the Z-inner-halo("garden ") (rule 3 and 11).

In (4c) we have the Medial verb passer (par) (to go through), which belongs to the Passer (par) class. This verb debotes a displacement from Z-outerhalo to Z-outer-halo, via Z-inner-halo, with respect to a location of reference. We here can apply both rules 9 and 10 (Medial verb and positional preposition), depending on which interpretation of the verb is chosen.

The rule 9 predicts that the location of reference has to be matched with the zone Z-inner-halo("garden "). We then conclude that the source is part of the Zouter-halo(Z-inner-halo("garden ")), ie. of the Z-outer-halo("garden ") (rule 1 and 9), that the path is part of the Z-inner-halo(Z-inner-halo("garden ")), ie. of the Zinner-halo("garden ") (rule 2 and 9) and that the goal is part of the Z-outer-halo(Zinner-halo("garden ")), ie. of the Z-outer-halo("garden ") (rule 3 and 9). That is, John is outside the garden before his motion; he enters the garden, crosses it and goes outside of it, in order to be outside the garden at the end of his motion.

The rule 10 predicts that the location of reference has to remain uninstantiated and that the goal has to be related to the Z-inner-halo("garden ") by a relation of spatio-temporal inclusion. We then conclude that the source is part of the Z-outer-halo(lref) (rule 1), that the path is part of the Z-inner-halo(lref) (rule 2) and that the goal is part of the Z-outer-halo(lref) (rule 3) and also part of the Z-innerhalo("garden ") (rule 10). That is, John is outside the garden before his motion, and moves in such a way that he finaly enters the garden in order to be inside the garden at the end of his motion. 


\section{A Comparative Survey of the Literature}

Finally, we have realized a comparative survey of the literature, distinguishing syntactic approaches ([Wun91], [Mai92], [Gui90],...) from semantic ones ([Hay89], [DB82], [Lam87], [Boo87], Lau91],...). Two conclusions emerge: syntactic approaches do not furnish completely precise criteria and do not cover all the so-called motion verbs, easily recognized with semantic approaches. Of course such approaches are not useless; in contrast, we think that syntactic approaches alone are not sufficient, but that they can bring some important elements to corrobate semantical criteria built on classifications. The second conclusion is important: all these works (including syntactic approaches) arrive at compatible classifications. This convergence of results for classifications built on so different kinds of criteria (syntactical, semantical, conceptual) gives to them a very strong validity. Our classification is compatible with all the others in the literature and moreover is more detailed.

\section{Conclusion}

We have presented along this paper a typology for motion verbs, spatial prepositions and motion complexes, built on a conceptual structuration of Space, which enables us to draw a detailed spatio-temporal semantics of motion expressions. The typology presented, especially for change of location verbs, is richer and more detailed than what is proposed in the literature. We have also shown, through our examples, that this richness, and the complexity which comes together with it, are needed in order to obtain correct and detailed spatio-temporal interpretation of motion descriptions.

The results presented here are used as basis for other investigations on motion in progress at the LRC (Language, Reasoning, Computation) research group of the IRIT, in Toulouse, France. We provide as examples an aspectual study of verbs of change of location for which the conceptual structuration of space, used here for the typology, leads to more detailed notions of the Aktionsart, and also linguistic comparative studies realized on Basquian (Michel Aurnague) and Japanese (Junichi Saito) languages which corroborate our own results for French.

\section{Endnotes}

* Thanks to Michel Aurnague, Mario Borillo, Andrée Borillo, Myriam Bras, Laure Vieu for helpful conversations on this topic.

\footnotetext{
${ }^{1}$ To do this, we must add some additional notions to the ontology developed by Vieu--in particular, we need a notion of frame of reference, which is given by a set of objects (locations and perhaps other objects) that are taken to be fixed. We then can compare the spatial position or spatial component of the STref of an object at two distinct times. But we will not go into details here. See [AS94].

2In [Ash93], the semantics of the dynamic properties is complicated by the fact that the DRS construction procedure generates all the different scope possibilities for quantifiers within a particular sentence. This requires that the predicative DRS A for a transitive verb be polymorphous in the sense that we can apply a subject partial DRS to A before we apply the partial DRS derived from the object NP. We will not worry about scope ambiguities here, and so we can take the syntax to completely specify the order of application of partial DRSs to predicative DRSs.
} 


\begin{abstract}
${ }^{3}$ See [Ash 93] for details and for the semantics of the partial/predicative DRS calculus. Without regard for scope ambiguities and anaphora resolution (which here may include specification of the Source and Goal of a particular motion complex), this process is completely compositional in the sense that the DRS is built up following the function argument structure implicit in the syntactic structure.

${ }^{4}$ This portion of space can be a bit of ground, such as in the square or in the lawn, or the space occupied and defined by an object, the inside of which is a cavity, such as in the house, or not, such as in the bench .

${ }^{5}$ They can only be lexicalized with deictic constructions like here, this position, the position Mary occupies now , ...

${ }^{6} \mathrm{We}$ define four and not only three classes of motion verbs because verbs of change of position do not all behave the same way. Compare for example : to run / to run in place and to circulate $/ *$ to circulate in place. This has led us to subdivise this class onto verbs of change of position (eg. to circulate) and verbs of inertial change of position (eg. to run).

${ }^{7}$ This ideal trajectory might be captured by means of an axiomatisation using nonmonotonic logic. This reminds us of elements of the English progresive in which similarly we appeal to "ideal" or "inertial" paths. With these progresive, such paths are naturally captured in a non-monotonic formalism [Ash92]. These ideal paths have the same properties as "normal" locations plus some others (like eg. a direction of motion and a stronger link with time), about which we will say nothing further in this paper.

${ }^{8}$ The relation of spatio-temporal inclusion used in our formalism is the part of relation $(\mathrm{P}(\mathrm{x}, \mathrm{y}))$ of the Calculus of Individuals of Clarke [Cla81], we have already talked about in section 2.

${ }^{9}$ Ie. in addition of the way already deduced from the rule 1.

${ }^{10}$ It has to be matched with some location given by the discourse (anaphora resolution
\end{abstract} [AW89]), by the context or the common shared world knowledge.

\title{
References
}

[AS94] Nicholas Asher and Pierre Sablayrolles. "A Typology and Discourse Semantics for Motion Verbs and Spatial PPs in French". To appear in a special issue of the Journal of Semantics on Theoretical Lexical Semantics, J. Pustejovsky.

[Ash94] Nicholas Asher. "Spatio-temporal Structure in Texts," in S. Akama ed. Festschrift for I. Onishi, Oxford University Press, in press.

[Ash92] Nicholas Asher. "A Truth Conditional, Default Semantics for the Progressive". Linguistics and Philosophy, No. 15, 1992.

[Ash93] Nicholas Asher. Reference to Abstract Objects in Discourse. SLAP Series vol. 50. Kluwer Academic Publishers.

[Ash-a193] Nicholas Asher, Michel Aurnague, Myriam Bras and Laure Vieu. "Space, Time and Discourse". Proceedings of the Workshop on Temporal and Spatial Reasoning, IJCAI-93.

[AV93] Michel Aurnague and Laure Vieu. "Toward a formal representation of space in language: A commonsense reasoning approach". Proceedings of the Workshop on Temporal and Spatial Reasoning, IJCAI-93.

[AW89] Nicholas Asher and Hajime Wada. "A Computational Account of Syntactic, Semantic and Discourse Principles for Anaphora Resolution". Joumal of Semantics, 1989.

[Aur91] Michel Aurnague. "Contribution à l'étude de la sémantique formelle de l'espace et du raisonnement spatial : la localisation interne en français, 
sémantique et structures inférentielles." $\mathrm{PhD}$ thesis, Université Paul Sabatier, Toulouse, France, February 1991.

[Boo85] Jean Paul Boons. "Preliminaires a la classification des verbes locatifs: les complements de lieu, leurs criteres, leurs valeurs aspectuelles". Linguisticae Investigationes, 9(2):195-267, 1985.

[Boo87] Jean Paul Boons. "La notion semantique de deplacement dans une classification syntaxique des verbes locatifs". Langue Francaise, 76:5-40, December 1987.

[Cla81] Bowman L. Clarke. "A Calculus of Individuals based on Connection". Notre Dame Journal of formal logic, 22(3):204-218, July 1981.

[Cla85] Bowman L. Clarke. "Individuals and Points". Notre Dame Journal of formal logic, 26(1):61-75, January 1985.

[Dav67] Donald Davidson. "The Logical Form of Action Sentences". Rescher, N. ed. The Logic of Decision and Action. Pittsburgh, PA: University of Pittsburgh Press.

[DB82] J. Dervillez-Bastuji. "Structure des relations spatiales dans quelques langues naturelles -- introduction a une theorie semantique". Library Droz, Geneva-Paris, 1982.

[Gui90] Alain Guillet. "Une classification des verbes transitifs locatifs". PhD thesis, Universite Paris 7, LADL, 1990.

[Gru65] J.S. Gruber. "Studies in Lexical Relations". Doctoral dissertation, MIT, 1965.

[Hay89] Ellen M. Hays. "On Defining Motion Verbs and Spatial Prepositions". Bericht B61, SFB 314: VITRA, Fachbereich 10 - Informatik IV, Saarbruecken, Germany, October 1989.

[Jac76] R.S. Jackendoff. "Towards an Explanatory Semantic Representation". Linguistic Inquiry, 7:89-150.

[Kam79] Hans Kamp. "Events, Instants and Temporal Reference". Semantics from different points of view, Eds R. Bauerle, U. Egli and A. Von Stechow, Springler Verlag, Berlin, pages 376-417, 1979.

[Lam87] B. Lamiroy. "Les verbes de mouvement, emplois figures et extensions metaphoriques". Langue francaise, 76:41-58, 1987.

[Lau91] Dany Laur. "Semantique du deplacement et de la localisation en francais: une etude des verbes, des prepositions et de leurs relations dans la phrase simple". PhD thesis, Universite Toulouse le Mirail, Toulouse, June 1991.

[Lau93] Dany Laur. "La relation entre le verbe et la preposition dans la semantique du deplacement". Language, La couleur des prepositions:47-67, June 1993.

[Les27-31] S. Lesniewski. "O podstawach matematyki". Przeglad filozoficzny No.30-40, 1927-31. Partial translation by G. Kalinowski in:

[Les89] S. Lesniewski. "Sur les fondements de la mathematique". Hermes, Paris, 1989.

[Mai92] Claudia Maienborn. "Kompakte Strukturen: Direktionale PPn und nichtlokale Verben". In P. Bosch and P. Gerstl eds., Sprachtheoretische Grundlagen fuer die Computerlinguistik, pages 131-145, DFG Sonderforschungsbereich 340, Stuttgart, Bericht Nr. 30, December 1992.

[Vie91] Laure Vieu. "Sémantique des relations spatiales et inférences spatiotemporelles : une contribution à l'étude des structures de l'espace en Langage Naturel. PhD thesis, Université Paul Sabatier, Toulouse, France, April 1991. 
[Wun91] Dieter Wunderlich. "How do prepositional phrases fit into compositional syntax and semantics?" Linguistics, 29:591-621, 1991. 